\title{
Tangence
}

\section{Le journal d'écrivain - œuvre d'imagination ou témoignage ? Sur le discours préfaciel}

\section{Jerzy Lis}

Numéro 45, octobre 1994

Authenticité et littérature personnelle

URI : https://id.erudit.org/iderudit/025831ar

DOI : https://doi.org/10.7202/025831ar

Aller au sommaire du numéro

Éditeur(s)

Tangence

ISSN

0226-9554 (imprimé)

1710-0305 (numérique)

Découvrir la revue

Citer cet article

Lis, J. (1994). Le journal d'écrivain - œuvre d'imagination ou témoignage ? Sur le discours préfaciel. Tangence, (45), 125-131. https://doi.org/10.7202/025831ar d'utilisation que vous pouvez consulter en ligne.

https://apropos.erudit.org/fr/usagers/politique-dutilisation/ 


\section{Le journal d'écrivain - œuvre d'imagination ou témoignage? Sur le discours préfaciel Jerzy Lis}

La problématique que nous nous proposons de traiter dans le présent article est celle du statut ambigu du journal d'écrivain. Nous avons choisi d'étudier plus particulièrement les journaux intimes écrits par des écrivains parce que, non seulement ceux-ci sont plus à même d'accorder de l'importance à la fonction esthétique de leur écriture, mais ce sont en majorité leurs journaux qui sont publiés et préfacés.

À mi-chemin entre le témoignage et les rêveries, le journal personnel reste difficile à cerner. L'étude du discours préfaciel de ce genre littéraire permet à cet égard d'apporter un éclairage intéressant.

L'idéal pour l'étude d'un journal serait de pouvoir évaluer la part qu'occupent dans les notes les fragments engendrés par l'imaginaire et les passages à caractère de témoignage. Mais, une telle démarche est utopique du fait même qu'il est impossible de tout dire et d'établir des contours nets entre l'événementiel et le subjectif. D'ailleurs, le diariste et le lecteur vivent, à la lecture d'un journal, chacun à sa propre manière une espèce d'illusion du vrai. Ni le lecteur ni même le diariste ne peut distinguer nettement où finit le témoignage et où commence l'invention.

La présentation du journal en tant qu'expression du témoignage est particulièrement visible au niveau du discours préfaciel. Il faut ici distinguer deux catégories de préfaces: auctoriale et allographe ${ }^{1}$. La première renvoie naturellement au diariste qui est lui-même l'auteur de l'introduction, de l'avertissement ou de l'avant-propos. L'écoulement du temps fait de lui un être plus sceptique, sinon plus indifférent à la nature de son témoignage. L'écho des circonstances dans lesquelles s'effectuait la notation est souvent mis en relief par le préfacier.

1 Pour la terminologie, voir Gérard Genette, Seuils, Paris, Seuil, 1987. 
Pour son journal tenu en 1916-1917, Paul Morand écrit son avant-propos seulement en $1947^{2}$. Il doute de la portée documentaire de ses notes car, à son avis, il n'y a là aucune révélation singulière sur des contemporains connus, ni de vue originale sur son époque. Trente ans plus tard les personnages décrits sont perçus par l'auteur "sous un jour souvent différent." Il en est de même pour l'écriture qui avec le temps lui apparaît "peu serrée" et les jugements "absolus". Mais il déclare n'avoir rien changé dans son texte. Cette intention de garder le texte intact caractérise aussi Paul Léautaud qui écrit dans l'avertissement du premier volume de son Journal littéraire:

Je n'ai ajouté, ni retranché un mot aux conversations, entretiens ou propos qu'on m'a rapportés ou que j'ai entendu tenir devant moi. [...] Les gens qui m'ont connu de près m'ont toujours tenu pour un homme qui ne ment jamais et, de plus, dénué de toute imagination $[\ldots] .^{3}$

En même temps le diariste ajoute au bas de la page une note du 24 septembre 1943 dans laquelle il signale avoir encadré par des lignes, des points, certains passages qui ne pouvaient être alors publiés. Paradoxalement ses aveux à propos des interventions dans le texte soulignent davantage la véracité de son témoignage même s'il apparaît incomplet aux lecteurs. Quant à Julien Green, à la fois diariste et préfacier, il tient à restituer dans la réédition des volumes de son journal les passages supprimés dans la version initiale. Dans Les années faciles (1916-1934), il réintègre, par exemple, certains commentaires politiques qui à ce moment précis (en 1970) avaient cessé d'être ridicules. Il s'est tout simplement rendu compte qu'ils contribuaient à reconstruire le climat de l'époque et pouvaient contenir un sens nouveau, nécessaire à une meilleure définition de soi. Autrefois ennuyants, de nombreux passages devenaient tout à coup intéressants. Léautaud et Green attachent une grande importance à une transcription fidèle de la réalité, le premier en vue d'obtenir l'image générale de la vie littéraire de son temps et le deuxième, celle de l'écrivain de son époque. La révision du texte d'autrefois ne sert qu'à confirmer la justesse des notes prises il y a bien des années.

2 Paul Morand, Journal d'un attacbé d'ambassade 1916-1917, Paris, Gallimard, 1963.

3 Paul Léautaud, Journal littêraire, vol. I (1893-1906), Paris, Mercure de France, 1954. 
Dans le cas où l'écart entre la notation et la publication n'est pas grand, la contestation de la valeur du texte, de la part du diariste, devient plus fréquente. Edmond Gilliard ${ }^{4}$ déclare avoir revu et adapté son texte aux "exigences de [sa] responsabilité d'aujourd'hui " tout en précisant qu'il use ainsi de son droit de propriété. Son cas n'est pas unique et l'on peut même constater une certaine régularité dans l'attitude des diaristes qui continuaient à tenir leurs journaux dans la période sombre de 1940 à 1945 . Face à toutes sortes d'interprétations politiques et morales de l'époque en question, la publication des notes personnelles de différents écrivains ne pouvait se faire sans la prise en considération d'éventuelles conséquences judiciaires et des persécutions morales.

La réflexion de Jules Roy sur la vanité et la prétention de ce procédé d'écriture "à une époque peu favorable à l'introspection " 5 n'est certainement pas dépourvue de sens. Ne pouvant s'exprimer autrement à cause de la guerre, le diariste s'était contraint à noter régulièrement ses impressions et, en général ses états d'âme, ceci pour ne pas mourir sans avoir témoigné. À la publication de ses notes conçues comme des pensées d'un condamné à mort et non pas comme celles d'un survivant, ont dû être triés et amputés des passages concernant des tierces personnes. Cependant il souligne que s'il avait pensé les publier un jour, elles auraient peut-être pris un tout autre caractère. Henry de Montherlant ${ }^{6}$, pour sa part, avoue avoir détruit certaines de ses notes relatives aux Allemands et à leur conduite pendant la guerre. Les remarques du type: "[notes] systématiquement hostiles aux nazis, écrites avec beaucoup de liberté, peu nombreuses et de peu d'intérêt", suffisent-elles à mesurer la valeur du témoignage perdu? Un autre diariste de l'époque, Jean Guéhenno, signale les lacunes du même ordre, mais fournit des explications plus précises: le lecteur ne doit pas s'attendre à trouver des faits inconnus ou secrets, parce que le diariste apporte son témoignage pour qu'un Français moyen puisse s'y reconnaître. N'ayant pas pu tout noter pour des raisons de sécurité, il a supprimé de

4 Edmond Gilliard, Journal, Lausanne, Mermod, 1945; son avertissement date du mois d'août 1945.

5 Jules Roy, Retour de l'enfer, Paris, Julliard, coll. "Voici Témoignages ", 1963; l'avant-propos date de 1951.

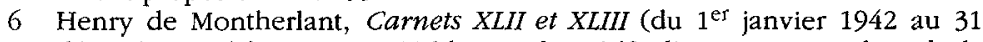
décembre 1943), Paris, La Table Ronde, 1948; l'avant-propos date de la même année. 
nombreuses pages de son manuscrit et rayé certains noms ${ }^{7}$. Malgré le parti pris spécifique à Guéhenno, son texte est un témoignage sur cette période sombre. En tout cas son Journal des années noires ne comprend aucun passage intime sur l'auteur.

L'analyse des prêfaces auctoriales mentionnées précédemment n'indique pas si, de manière générale, l'écrivain considère avant tout son journal par rapport à sa fonction documentaire. Les renseignements donnés et les restrictions dues à l'auto-censure rendent le document mutilé pour ce qui est de sa valeur dite historique et les diaristes eux-mêmes ne le cachent pas. Il est évident que les omissions, les coupures ou toutes sortes de silences voulus dans le texte informent également sur bien des choses. Les coupures sont justifiées et moins douloureuses pour le diariste si la publication du texte est prévue d'avance. $\mathrm{Si}$, au contraire, le texte est écrit pour soi, il peut à l'inverse engendrer une écriture beaucoup plus libre et sincère, ce dont témoignent les propos de Green dans l'une de ses préfaces (il s'agit de la réédition du troisième volume de son journal Devant la porte sombre qui embrasse les années 1940-1943).

Le discours préfaciel de Denis de Rougemont dans son Journal d'une époque mérite une attention particulière. L'auteur y a réuni plusieurs journaux écrits entre 1926 et 1946, publiés séparément déjà bien avant $1968^{\circ}$. Outre la préface générale du volume, le lecteur trouve aussi deux avertissements, placés respectivement en tête du Joumal d'Allemagne et du Journal des deux mondes, qui ont trait à une espèce de théorie du journal. Denis de Rougemont fait la distinction entre l'intime et le privé et place son journal ( qui comprend son "journal non intime", et son "journal privé") à distance égale "de la chronique impersonnelle et de la confidence non sans vue de publier". Il pense que pour décrire un événement on peut soit l'intérioriser, soit le projeter sous le masque d'une relation objective. L'interaction du témoignage et de l'écriture lui paraît indispensable, faute de savoir ce qu'il reste finalement de "vrai " après l'acte de transcrip-

7 Jean Guéhenno, Journal des années noires (1940-1944), Paris, Gallimard, 1973 (1 re éd. 1947).

8 Denis de Rougement, Joumal d'une époque (1926-1946), Paris, Gallimard 1968. Le volume comprend: Le paysan de Danube (1926-1929), Journal d'un intellectuel en chomage (1933-1935), Journal d'Allemagne et Journal des deux mondes (1939-1946). 
tion. Ses notes espacées sur une vingtaine d'années ont pour but de traduire la réalité d'une époque qui prend fin sous ses yeux. "Il se peut que le journal privé soit la forme de transition qui corresponde à la réalité d'un temps nouveau: elle traduit les relations d'une personne avec les passions collectives". Même si les confidences strictement personnelles sont évacuées dans un journal "intime" qu'il avoue tenir, il lui est impossible de dissimuler ou de passer sous silence les descriptions ou impressions qui le mettent en relation avec les événements décrits. "Finalement, écrit-il, quoiqu'on dise on dit tout, si le lecteur a l'oreille fine". Il déclare être fait pour écrire une "chronique des moments de présence à moi-même et au monde conjointement ", formule qui apprend au lecteur beaucoup plus que telle ou telle définition du journal. La clarté du projet diariste de Rougemont a évidemment pour but d'éviter toute interprétation indésirable du texte de la part du lecteur. Quant à Henry de Montherlant, il prévient le lecteur, dans l'une de ses préfaces, qu'il se trompe s'il s'attend à trouver dans ce volume publié le compte rendu de ses préoccupations pendant la période de l'avant-guerre. Il a décidé de retirer tous les textes et tous les sujets qui l'avaient alors préoccupé 9 .

Si la préface écrite par le diariste lui-même valorise nettement les intentions très personnelles de son journal et met en relief le besoin d'offrir au lecteur un texte relativement bien construit, celle préparée par des tiers (famille du diariste, ami ou éditeur) en accentue surtout la valeur documentaire, humaine ou artistique. Écrite en principe après la mort du diariste, la préface allographe authentique sert à prouver au lecteur qu'il détient entre ses mains un texte unique et exceptionnel dont la valeur n'est pas contestable. Une telle publication a pour but, le plus souvent, de compléter la biographie et l'œuvre de l'écrivain en question par la présentation de la richesse de sa vie intellectuelle et de ses préoccupations. Il n'est pas toujours sûr que le diariste aurait souhaité que son texte fût publié sous la forme que nous proposent les éditeurs, tout comme d'ailleurs, on ne peut pas toujours savoir si de son vivant l'écrivain aurait exprimé la volonté de divulguer ses écrits personnels. Rares sont les cas où la préface se limite à la simple présentation du texte qui va suivre. C'est pourtant le cas de Philippe Barrès qui exécute la dernière volonté de son père,

9 Henry de Montherlant, Carnets XXIX à XXXV (du 19 février 1938 au 11 janvier 1939), Paris, La Table ronde, 1947; préface datée de 1947. 
Maurice, en publiant une édition posthume de ses Cabiers. Selon ses dires aucune altération ni addition n'a été faite. Pour assurer la véracité des cahiers, on a même reproduit fidèlement la disposition du manuscrit et ajouté un aperçu de circonstances ainsi qu'un résumé biographique ${ }^{10}$. L'intention de Robert Mallet, préfacier de Larbaud, est d'une autre nature. Éditeur des ouvres complètes et détenteur de ses archives, Mallet juge nécessaire de préserver de l'oubli les pages qui, selon lui, n'ont aucun intérêt sur les plans psychologique et littéraire. Il limite leur importance au seul usage des historiens de la littérature et des spécialistes de Larbaud. Et pourtant, malgré cette promesse il n'offre aux lecteurs qu'une sélection de notations ${ }^{11}$.

De nombreux préfaciers tiennent à souligner l'authenticité du document. Juliette Du Bos parle de la "parfaite bonne foi " et de la "prodigieuse mémoire" de son mari Charles et atteste ainsi la véracité du journal ${ }^{12}$. Les éditeurs de Jules Roy font la même chose d'une manière un peu différente. D'ailleurs la note qui suit ne laisse pas de doutes sur l'authenticité du témoignage: "On y suivra, presque heure par heure, cette lutte épuisante et dévastatrice qu'était devenue la guerre industrielle dans les airs " 13 .

Il importe d'aborder succinctement maintenant un autre aspect du discours préfaciel: les références du préfacier à des parties du journal qui ont été, pour une raison ou pour une autre, supprimées lors de la publication. Probablement seul à connaître les lacunes le préfacier - auteur ou non du journal - a souvent tendance à énumérer les rubriques habituelles du diariste pour faciliter sinon diriger la lecture du texte. Ainsi ces quelques exemples assez significatifs: Gérard-Bailly a renoncé à inclure dans le

10 Maurice Barrès, Mes cabiers, vol. I (janvier 1896-février 1898), Paris, La Palatine, 1929; préface de Philippe Barrès.

11 Valêry Larbaud, Journal 1912-1935, Paris, Gallimard, 1955; introduction par Robert Mallet. Dans le neuvième volume des CEuvres complètes de Larbaud qui comprend le Journal inédit (Gallimard, 1954), le préfacier parle du choix: "Valéry Larbaud a bien voulu me confier les Cabiers qu'il n'a pas détruits, en me demandant d'y faire un choix basé sur l'intérêt plus ou moins grand de ses notations. "

12 Charles Du Bos, Journal, vol. IV (l'année 1928), Paris, Corréa, 1950; préface par Juliette Du Bos.

13 Il faut préciser que cette note des éditeurs fait bien partie du péritexte, mais la préface dont j'ai parlé plus haut est écrite par le diariste lui-même. $C f$. Jules Roy, Retour de l'enfer, op. cit. 
texte des notes qui auraient pu être bien intéressantes du point de vue du document humain: "faits, incidents, impressions de voyage, paysages, portraits, pensées, rêveries, confessions, jugements, prix de vin, plan de table et d'invités, prescription médicale, adresse ou les horaires de trains " lesquelles se trouvaient parsemées entre des pages de réflexions ou de plaintes graves. Si l'on insiste sur le témoignage pourquoi supprimer alors, comme c'est le cas dans les Carnets de Saint-Exupéry, de nombreuses informations d'ordre économique ${ }^{14}$ ?

Il faudrait ajouter enfin que la décision de publier le journal enferme une contradiction en soi. Toute marque typographique participe à l'authenticité du témoignage. La description des cahiers, leur format et leur couleur, tout ce côté fétichiste et technique de l'activité diariste n'est pas non plus sans importance pour la considération du texte. Évidemment il arrive souvent que l'intervention de l'éditeur dans le contenu du journal enlève au texte une partie du naturel et du spontané. Je ne parle pas de cas extrêmes de censure faite par le préfacier et le rédacteur en personne dans les passages où il est lui-même impliqué. Il me vient ici à l'esprit Marie Dormoy et son intransigeance pour bien des pages de Léautaud.

La publication du journal change non seulement l'attitude créatrice du diariste mais aussi sa position de témoin. Si le diariste se présente souvent comme un observateur sincère, il n'empêche qu'il ne peut évacuer complètement de ses écrits toute forme de subjectivité. D'autre part, seule la confrontation entre le texte publié et le manuscrit peut permettre d'évaluer et de juger en quoi les remarques notées sur le coup diffèrent de la version livrée aux lecteurs. Une version première et retravaillée ultérieurement constitue une perception du réel transformée soit par une nouvelle évaluation de ce "réel", soit par un souci d'amélioration de style.

Mais, si une étude intertextuelle entre version manuscrite et version publiée constitue une démarche souvent nécessaire à l'étude des journaux d'écrivains, celle des préfaces auctoriales dévoile l'inéluctable écart entre le réel vécu, le réel retranscrit et le réel revisité par une lecture ultérieure. Quant aux préfaces allographes, elles ont le plus souvent tendance à faire écran au réel rapporté par le diariste à cause de leur interaction sur les lecteurs.

14 Antoine de Saint-Exupéry, Camets, Paris, Gallimard, 1975; préface de Pierre Chevrier. 